Rev. Biol. Trop., 47(1-2): 189-201, 1999

www.ucr.ac.cr www.ots.ac.cr www.ots.duke.edu

\title{
Diversidad de hormigas (Hymenoptera: Formicidae) en un gradiente sucesional del bosque nublado (Nariño, Colombia)
}

\author{
Catalina Estrada M. ${ }^{1}$ y Fernando Fernández C. ${ }^{2}$ \\ 1 Instituto de Genética, Universidad de los Andes, Apartado Aéreo 4976 Santafé de Bogotá, Colombia. Fax 571- \\ 2841890 catalinaestra72@hotmail.com \\ 2 Instituto de Investigación de Recursos Biológicos 'Alexander von Humboldt', Apartado Aéreo 8693, Santafé de \\ Bogotá, Colombia.
}

Recibido 14-V-1998. Corregido 27-XI-1998. Aceptado 7-XII-1998

\begin{abstract}
We evaluate the potential use of ants as indicator taxa of cloud forest recovery in La Planada Reserve, at southwestern Andine slope in Nariño, Colombia. The ants were sampled from February through August 1995, using pitfall traps, tuna baits and manual collection, in seven localities representing forest successional stages: cattle ground, grassland (three year old), two ten year old forests and 20 year old, exploit and primary forests. We found 63 species ( 29 genera) with a 20-26 species richness per locality, with the exception of the ten year old forest (12 species). There were no any statistical differences in species richness and diversity among samples and study sites. Nevertheless taxonomic composition was associated with the successional stage (Simple Matching Coefficient). Ants can be used as indicators of forest recovery at La Planada.
\end{abstract}

Key words: Colombia, ants, indicator species, successional stages, disturbance, cloud forest.

Los bosques montanos y premontanos de los Andes Tropicales, a pesar de ocupar sólo $0.2 \%$ del planeta (Ortiz von Halle 1991), son los mayores centros de endemismo y diversidad biológica del mundo (Anónimo 1986). Gran parte de esta diversidad biológica se pierde por el acelerado crecimiento de la población así como de las actividades industriales y agrícolas, que han ocasionado la devastación del 90\% de ellos (Anónimo 1986, Ortiz von Halle 1991). Uno de los grupos de animales menos conocidos dentro de estos bosques son los insectos, además son el componente más diverso de los ecosistemas terrestres (Kim 1993, Kremen et al. 1993) y a pesar de esto, su conservación ha sido un subproducto del esfuerzo de conservación de plantas y vertebrados y no una meta central de dichas políticas (Janzen 1987, Kim 1993, Miller 1993).
Una cualidad que podría resaltar la importancia de los insectos para el hombre es la capacidad de servir como indicadores ecológicos (Brown 1991, Holloway \& Stork 1991, Kremen et al. 1993, Samways 1993). Una especie o grupo de especies indicadoras son organismos cuyas características (presencia o ausencia, densidad de población o dispersión), son utilizadas para obtener información sobre el estado de la calidad del ambiente (Landres et al. 1988). Pearson (1994) resume en siete puntos los principales criterios biológicos y logísticos para escoger indicadores eficientes: (1) taxonomía bien conocida y estable que permita que las poblaciones sean bien definidas, (2) historia de vida y biología bien conocidas, (3) fácil observación y manipulación en el campo, (4) amplio rango geográfico y alta diversidad taxonómica y ecológica, (5) especialización 
dentro de un hábitat restringido y sensibilidad a cambios en el hábitat, (6) evidencia de que los patrones que siga el taxon indicador se reflejen en otros táxones y que la respuesta a la perturbación sea predecible, rápida, analizable y lineal; y por último, (7) que los táxones escogidos puedan tener importancia económica para facilitar la realización y financiación de proyectos.

Las hormigas son uno de los grupos de insectos que cumplen con las condiciones requeridas para reflejar lo que sucede a otros grupos en ambientes cambiantes o para detectar centros de concentración de especies raras y endemismos (Majer 1983, Andersen 1990, Brown 1991). Algunos trabajos que relacionan la distribución de especies de hormigas con gradientes sucesionales o usos diferentes de la tierra han concluido que, con el aumento en la complejidad estructural del ecositema también aumenta la riqueza y diversidad de especies (e.g. Room 1975, Majer \& Camer-Pesci 1991, Roth et al. 1994, Bustos \& Ulloa-Chacón 1996-1997). En otros estudios, por el contrario, las diferencias de estos parámetros en los lugares de estudio no han sido tan claras, no se han encontrado o no muestran tendencias similares con otros grupos de animales (Belshaw \& Bolton 1994, Lawton et al. 1998), sugiriendo la necesidad de seguir realizando estudios locales que proporcionen más información sobre las relaciones entre las especies de hormigas y los ecosistemas naturales y modificados.

Este estudio aporta información sobre la distribución de especies de hormigas en los ecosistemas de montaña, como una forma de conocer y ayudar a conservar su diversidad biológica. Se comparan según su fauna de hormigas diferentes sitios de muestreo correspondientes a un gradiente sucesional en un bosque nublado, con el fin de buscar parámetros ecológicos o grupos de especies indicadoras que sirvan posteriormente para una evaluación del grado de perturbación o recuperación de dichos bosques.

\section{MATERIALES Y MÉTODOS}

El trabajo se llevó a cabo en la Reserva Natural La Planada, ubicada en la vertiente oc- cidental de la Cordillera de los Andes $\left(1^{\circ} 5^{\prime} \mathrm{N}\right.$, $\left.77^{\circ} 24^{\prime} \mathrm{W}\right)$. Comprende 3200 ha de bosque húmedo premontano (sensu Holdridge, Anónimo 1977), la mayor parte ubicada a una altitud de $1850 \mathrm{~m}$. Presenta una precipitación promedio anual de 4742 mm (1986-1995) distribuida bimodalmente con un periodo seco entre junio y agosto. Los ámbitos de temperatura comprenden desde $13.2{ }^{\circ} \mathrm{C}$ (mínima diaria promedio) hasta $25.3{ }^{\circ} \mathrm{C}$ (máxima diaria promedio). La Planada fue fundada por el Fondo Mundial para la Naturaleza (WWF) y la Fundación FES (Colombia) en 1983 y actualmente presenta bosques con diferentes grados de recuperación debido a que allí se practicó la explotación de maderas finas y la ganadería hasta antes de su fundación.

El trabajo se realizó en dos fases de campo, la primera entre los meses de febrero y marzo y la segunda entre junio y agosto de 1995. Para el propósito del estudio se escogieron siete localidades que representan un gradiente sucesional y cuyas características aparecen en el Cuadro 1.

En cada localidad se trazaron cuatro transectos paralelos de 100 m cada uno y separados entre sí $30 \mathrm{~m}$. Cada $10 \mathrm{~m}$ se ubicó una trampa de caída (pitfall) y una trampa con atún como cebo en el estrato arbóreo (a la altura del pecho). Además se colocaron por cada localidad ocho trampas de cebo en el estrato hipógeo. Esto para un total de 96 trampas por sitio por muestreo. Las trampas de cebo fueron revisadas dos veces al día y su contenido recolectado. Estas trampas al igual que las pitfall fueron recogidas después de 72 horas y su contenido fue conservado de manera individual (por trampa) en alcohol al 70\%. Estos muestreos se complementaron con colección manual y colección de hojarasca realizada durante dos horas diarias, para un total de seis horas por tipo de bosque. Colecciones de referencia del material se depositaron en el Instituto Humboldt (Villa de Leyva, Colombia) y en La Reserva Natural La Planada (Nariño, Colombia).

Se midieron además otras variables que se ha demostrado afectan la abundancia y diversidad de insectos (Levings 1983, Torres 1984a y 


\section{CUADRO 1}

Sitios de muestreo que representan un gradiente sucesional

Sitios de muestreo
Potrero (P)
Pastizal $(\mathrm{Pa})$

Bosque de 10 años (10a)

Bosque de 10 años (10b)

Bosque de 20 años (20)

Bosque entresacado (E)

Bosque maduro (M)
Estado sucesional

Utilizado actualmente para el pastoreo de ganado vacuno tres periodos al año.

Regenerado a partir de potrero hace 3 años.

Regenerado a partir de potrero hace 10 años.

Regenerado a partir de potrero hace 10 años. Más desarrollado que el lugar anterior.

Regenerado a partir de potrero hace 20 años.

Utilizado para la extracción selectiva de maderas finas hasta hace 15 años.

Ha tenido muy poca intervención humana

\author{
Características \\ Cubierto de pasto hasta de $1.5 \mathrm{~m}$.
}

Cubierto de pasto de 0.20 a $1 \mathrm{~m}$ de altura y algunos árboles de Clusia y Miconia.

Suelo cubierto de musgo y Lycopodium sp. Domina Killipiella steriofila, Vismia ferruginea, Tibouchina lepidota, especies de Clusia y de Miconia.

Domina T. lepidota, V. ferruginea, especies de Clusia y de Miconia

Domina $V$. ferruginea, especies de Clusia, Miconia, Faramea, Palicourea, Hyeronina y de Elaeagia.

Sobresalen especies de Prestoea, Ficus, Chamaedorea, Siparuna, Otoba, Macrolobium, Critoniopsis, Cyathea y de Elaeagia.

Sobresalen especies de Elaeagia, Hyeronina, Otoba, Quararibea, Cyathea, Nectandra, Sluanea, Inga, Otoba y de Prestoea. 1984b, Andersen 1990) y ayudan a caracterizar mejor los lugares de estudio. Estas variables son:

- Temperatura a nivel del suelo: Se midió colocando un termómetro de temperaturas máximas y mínimas en cada transecto durante los tres días de muestreo de cada sitio. La temperatura máxima y mínima de cada lugar de estudio es el promedio de medidas entre los cuatro transectos y los tres días de muestreo en cada fase de campo.

- Cantidad de hojarasca: Se tomó el peso seco promedio de seis muestras de hojarasca colectadas al azar en cada sitio de muestreo. Se utilizó una cuadrícula de $25 \mathrm{~cm}$ de lado (Roth et al. 1994).
- Heterogeneidad del hábitat: Para cada localidad se realizaron dos transectos de vegetación de 30 por $3 \mathrm{~m}$. En estos transectos se midió el DAP (diámetro a la altura del pecho) y la altura de todos los árboles y arbustos ubicados dentro del área demarcada. Los valores de DAP fueron agrupados en rangos de $5 \mathrm{~cm}$ y la altura en rangos de $2.5 \mathrm{~m}$. Con los valores de DAP se obtuvo un Indice de Heterogeneidad Horizontal del hábitat para cada sitio de muestreo, usando las clases diamétricas como especies y adaptándose el Indice de Diversidad de Simpson (Ludwing \& Reynolds 1988, Magurran 1988, Bustos \& Ulloa-Chacón 1996-1997). 


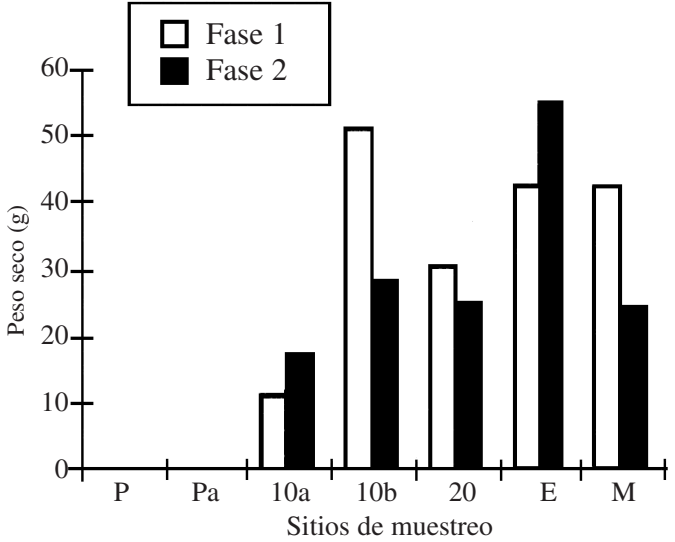

Fig. 1. Peso seco de hojarasca medido en cada sitio de muestreo. $\mathrm{P}$ potrero, Pa pastizal, 10a y $10 \mathrm{~b}$ bosques de 10 años, 20 bosque de 20 años, E bosque entresacado y M bosque maduro.

Para cada localidad de muestreo se calculó la riqueza (expresada en número de especies de hormigas), se hallaron los índices de diversidad y equitabilidad de Shannon y se estimó el número de especies abundantes (N1) y muy abundantes (N2) con los números de diversidad de Hill (Ludwing \& Reynolds 1988). Se utilizó la frecuencia de captura como un parámetro de abundancia relativa (Romero \& Jaffé 1989). Las hormigas recolectadas con el método de colección manual no se incluyeron en el análisis de diversidad y equitabilidad.

Para comparar las poblaciones de hormigas entre los diferentes sitios de muestreo se utilizó el coeficiente de asociación Simple Matching analizado con el Método de Ligamiento Promedio (UPGMA) (Crisci 1983).

\section{RESULTADOS}

Con excepción del promedio de peso seco de hojarasca (Fig. 1), los parámetros que se midieron para caracterizar los lugares de estudio mostraron tendencias relacionadas con el gradiente sucesional. El Indice de Heterogeneidad Horizontal disminuyó con el aumento en la sucesión lo que corresponde a una mayor heterogeneidad horizontal (Fig. 2). Para este índice el

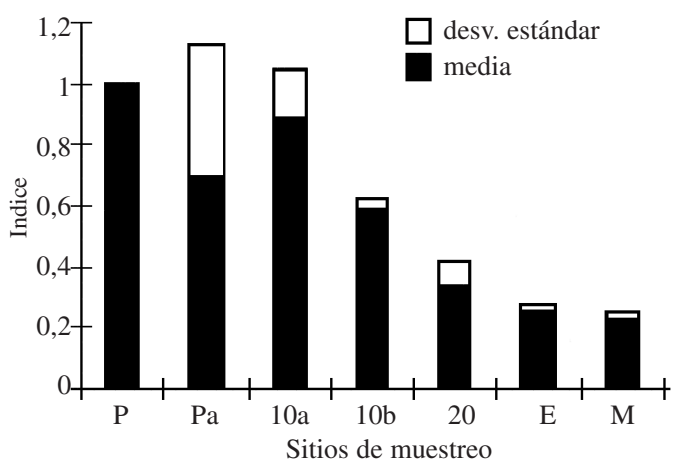

Fig. 2. Indice de heterogeneidad horizontal medido para cada sitio de muestreo. P potrero, Pa pastizal, 10a y $10 \mathrm{~b}$ bosques de 10 años, 20 bosque de 20 años, E bosque entresacado y $\mathrm{M}$ bosque maduro.

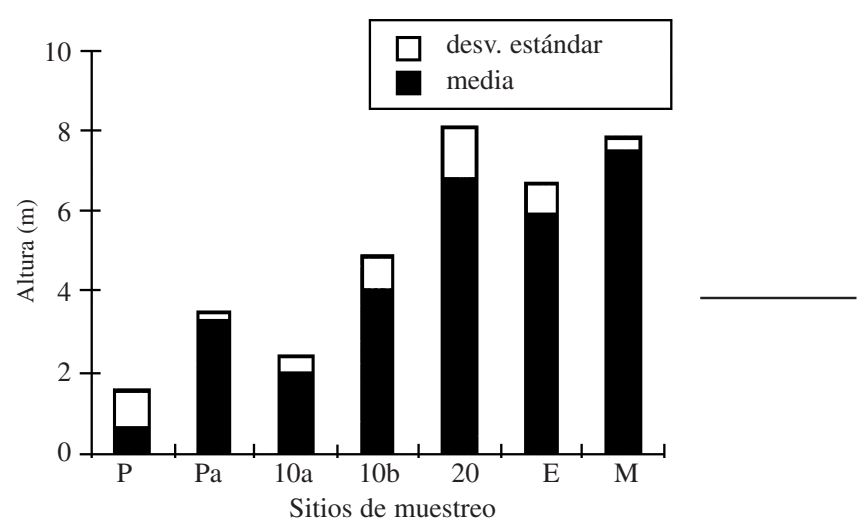

Fig. 3. Altura promedio de los árboles en las parcelas de cada sitio de muestreo. P potrero, Pa pastizal, 10a y 10b bosques de 10 años, 20 bosque de 20 años, E bosque entresacado y $\mathrm{M}$ bosque maduro.

pastizal $(\mathrm{Pa})$ exhibió un valor mayor al que se esperaba, desviándose de la tendencia. Esto puede atribuirse, en parte, a la presencia de árboles aislados que fueron conservados en el pastizal, algunos de los cuales quedaron incluidos en los transectos de vegetación. La altura promedio de los árboles apoyó lo encontrado con el índice de heterogeneidad, presentándose, como es de esperarse, mayores alturas a medida que el bosque era más recuperado o bien conservado (Fig. 3). Con respecto a la temperatura a nivel del suelo, los valores mostraron que la diferencia entre la temperatura mínima y máxima tiende a disminuir a medida 

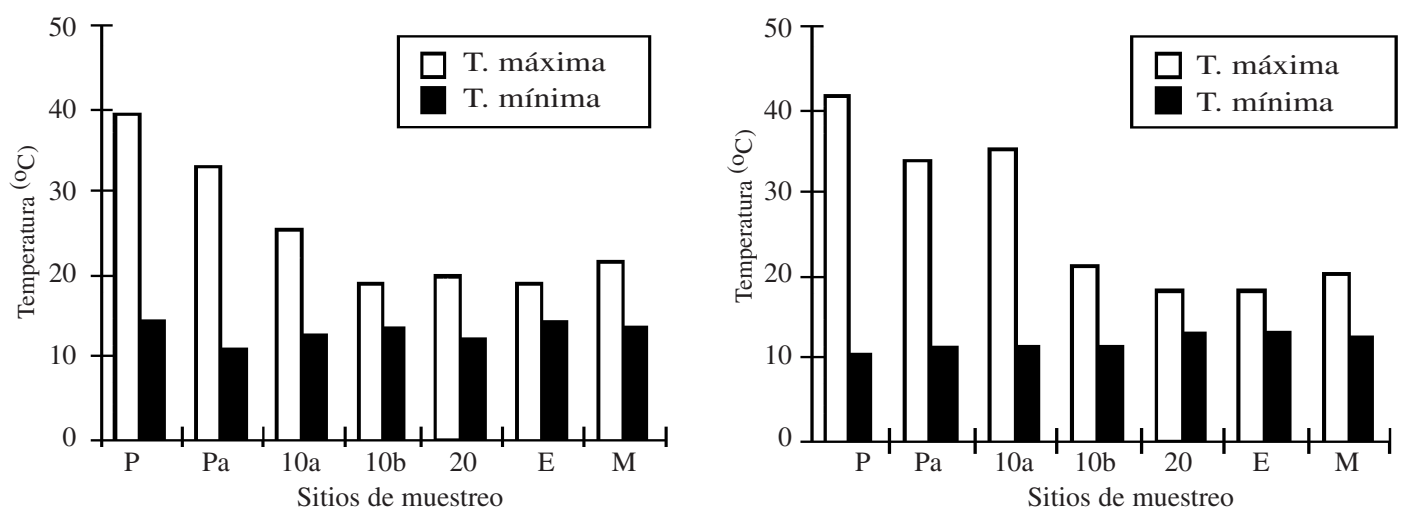

Fig. 4. Temperatura máxima y mínima medidas a nivel del suelo en cada lugar de estudio. P potrero, Pa pastizal, 10a y 10b bosques de 10 años, 20 bosque de 20 años, $\mathrm{E}$ bosque entresacado y $\mathrm{M}$ bosque maduro.

que se avanza en el gradiente sucesional (Fig. 4). Se encontró que por su parte, la temperatura máxima disminuye con el aumento en el desarrollo de la vegetación, con diferencias significativas entre sitios (ANOVA dos vías $\mathrm{P}<0.0001$ ) y entre fases de campo (ANOVA dos vías $\mathrm{P}<0.0438$ ) (Fig. 4). La temperatura mínima, aunque menos variable, mostró diferencias significativas entre lugares de estudio (ANOVA dos vías $\mathrm{P}<0.0001$ ) y entre fases de campo (ANOVA dos vías $\mathrm{P}<0.0001$ ), con una tendencia a ser mayor a medida que se avanza en el gradiente sucesional (Fig. 4). El promedio de peso seco de hojarasca resultó diferente entre lugares de estudio (ANOVA dos vías $\mathrm{P}<0.0001$ ) pero no entre fases de campo (ANOVA dos vías $\mathrm{P}<0.2830$ ). Al parecer la abundancia de hojarasca está determinada más por la presencia o ausencia de un dosel cerrado que por el tiempo de recuperación del bosque (Fig. 1). Esto se sugiere a partir de observar que los lugares con promedios de peso seco ligeramente superiores fueron el bosque de 10 años (10b) y el entresacado (E), que están alejados en el gradiente sucesional (Prueba de Duncan).

Se colectó un total de 63 especies de hormigas, pertenecientes a 29 géneros y 5 subfamilias (anexo), entre las que se destaca un nuevo género y nueva especie descrito para Myrmicinae, Lenomyrmex mandibularis (F. Fernández \& E. Palacio en prensa). La rique- za de hormigas fue muy similar entre los lugares de muestreo, presentando valores entre 20 (bosque de 10 años -10a) y 26 especies (bosque entresacado -E), con excepción del bosque de 10 años (10b) en el cual solo se hallaron 12 especies (anexo). Los índices ecológicos calculados para comparar la fauna de hormigas entre lugares con diferente grado de recuperación, no mostraron diferencias significativas ni entre sitios, ni entre fases de campo (Cuadro 2). La única diferencia fue registrada para el índice de equitabilidad de Shannon (ANOVA dos vías $\mathrm{P}<0.0457$ ) la cual, según una prueba de Duncan, se origina por un valor promedio de este índice un poco mayor en el bosque entresacado (E).

Una relación entre los lugares de muestreo y las especies encontradas en ellos aparece en el anexo. Sobresalen algunas especies dominando en todos los lugares como: Pachycondyla apicalis, Gnamptogenys sp. 1, Pheidole sp. 4 (excepto en E) y Camponotus sp. 1 (excepto en 20 y M); otras especies comunes en los lugares menos recuperados como: Pheidole sp. 1 (de P a 20), Procryptocerus sp. (de P a 10b) y Linepithema sp. 1 (de P a 20 excepto en 10b); y especies de hormigas comunes en los sitios mejor conservados como Megalomyrmex sp. 1 (en 20 y E) y Pachycondyla carbonaria (en M) (esta especie fue reportada en bosques intervenidos del Valle del Cauca, Colombia, P. Chacón - Com. Pers.). 


\section{CUADRO 2}

Indices de diversidad medidos para los sitios de muestreo (promedio \pm desviación estándar). Las dos últimas filas presentan los resultados de ANOVAS de dos vías realizadas para cada variable

Sitios de Muestreo

Potrero (P)

Pastizal (Pa)

Bosque 10 años (10a)

Bosque de 10 años (10b)

Bosque de 20 años (20)

Bosque entresacado (E)

Bosque maduro (M)

Diferencia entre lugares

Deferencia entre muestreos

\begin{abstract}
Riqueza
\end{abstract}
$17.5 \pm 2.12$

$18.5 \pm 4.95$

$17 \pm 0$

$9 \pm 2.83$

$15.5 \pm 2.12$

$18.5 \pm 3.53$

$17 \pm 1.14$

$\mathrm{P}<0.095 \mathrm{~ns}$

$\mathrm{P}<0.207$ ns

Diversidad

$1.48 \pm 0.15$
$1.61 \pm 0.04$
$1.38 \pm 0.06$
$0.95 \pm 0.18$
$1.51 \pm 0.11$
$1.68 \pm 0.59$
$1.1 \pm 0.62$
$\mathrm{P}<0.4649 \mathrm{~ns}$
$\mathrm{P}<0.8829 \mathrm{~ns}$

$\mathrm{P}<0.8829 \mathrm{~ns}$
Equitabilidad

$0.64 \pm 0.02$

$0.62 \pm 0.01$

$0.58 \pm 0.01$

$0.51 \pm 0$

$0.71 \pm 0.02$

$0.79 \pm 0.01$

$0.5 \pm 0.18$

$\mathrm{P}<0.0457^{*}$

$\mathrm{P}<0.4289$ ns
N1

$4.39 \pm 0.66$

$4.97 \pm 0.18$

$4 \pm 0.26$

$2.59 \pm 0.44$

$4.54 \pm 0.50$

$5.79 \pm 3.20$

$3.29 \pm 1.92$

$\mathrm{P}<0.5471^{\mathrm{ns}}$

$\mathrm{P}<0.9119$ ns
N2

$3.02 \pm 0.04$

$3.26 \pm 0.13$

$2.78 \pm 0.09$

$1.82 \pm 0.22$

$3.53 \pm 0.19$

$4.31 \pm 1.85$

$2.15 \pm 1.07$

$\mathrm{P}<0.2386^{\mathrm{ns}}$

* Probabilidad menor al 5\%, ns no significativo

Aunque la riqueza de especies de hormigas en los diferentes sitios de muestreo es similar, la composición varía de un lugar a otro, siendo mayor la diferencia entre más alejados estén en el gradiente sucesional. El dendrograma de similitud Simple Matching que tiene en cuenta la ausencia-presencia de las especies de hormigas, relacionó de forma satisfactoria los sitios con respecto a su grado de recuperación (Fig. 5). Se forman en este árbol tres grupos; el primero, con un grado de similaridad mayor conformado por el potrero y el pastizal (P y $\mathrm{Pa})$, sitios que además de ser parecidos en su vegetación, tienen la menor distancia (en tiempo) de recuperación (aproximadamente 3 años). El segundo grupo está formado por los dos bosques de 10 años, que aunque tienen la misma edad pueden considerarse más distanciados que los anteriores por la cercanía que tiene cada uno de ellos a bosques que promueven su recuperación. Por último, y menos relacionado, se encuentra la asociación de bosque de 20 años y bosque entresacado (E), lugares sometidos a diferente perturbaciones. El bosque maduro (M), según su composición de hormigas, es muy diferente a los otros lugares de muestreo.

Algunas especies fueron escogidas porque su presencia podría servir para evaluar el grado de recuperación del bosque donde se encuentran. Estas especies fueron seleccionadas utilizando como criterio que exhibieran alguna tendencia con respecto a preferencia de hábitat

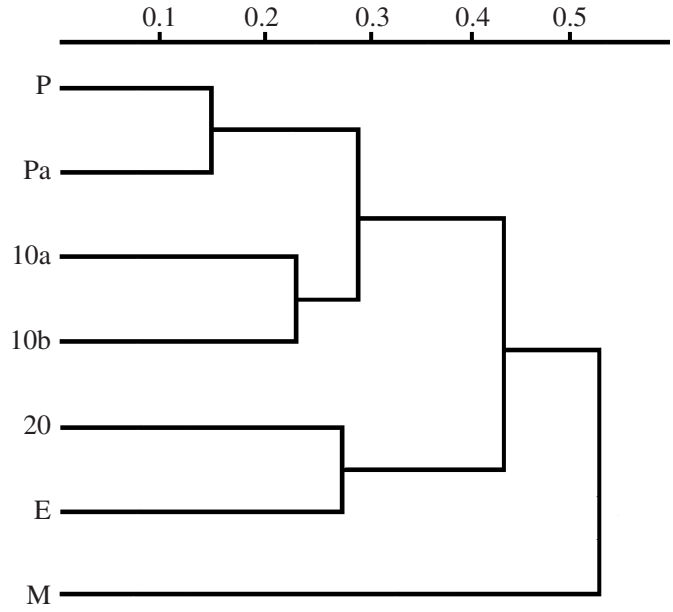

Fig. 5. Dendrograma de similitud entre sitios de muestreo según ausencia o presencia de especies de hormigas. Coeficiente de asociación Simple Matching y UPGMA. P potrero, Pa pastizal, 10a y 10b bosques de 10 años, 20 bosque de 20 años, $\mathrm{E}$ bosque entresacado y $\mathrm{M}$ bosque maduro.

y que aparecieran en las dos fases de campo (preferiblemente más de una vez por cualquier método de colección) (Cuadro 3). De esta manera se pueden identificar tres grupos de hormigas. El primero formado por Myrmelachista sp. 2 y Procryptocerus sp., quienes prefieren los lugares que han tenido menos tiempo de recuperación (del potrero a los bosques de 10 años). Un segundo grupo está formado por la especie Pheidole sp. 1, la cual además de estar presente en todos los lugares que alguna vez 


\section{CUADRO 3}

Especies que se proponen como indicadoras del grado de recuperación del bosque en la Reserva Natural La Planada

$\begin{array}{lll}\text { Especie } & \mathrm{P} & \mathrm{P} \\ & & \\ \text { Myrmelachista } \text { sp. } 2 & \mathrm{X} & \mathrm{X} \\ \text { Procryptocerus } \text { sp. } & \mathrm{X} & \mathrm{X} \\ \text { Pheidole } \text { sp. } \text { 1 } & \mathrm{X} & \mathrm{X} \\ \text { Camponotus abdominalis } & & \\ \text { Myrmelachista } \text { sp. } 1 & & \\ \text { Megalomyrmex } \text { sp. } 1 & & \\ \text { Pheidole } \text { sp. } \text { 5 } & & \\ \text { Hypoponera } \text { sp. } 2 & \\ \text { Pheidole } \text { sp. } 6 & \\ \text { Pachycondyla carbonaria } & \end{array}$

fueron potrero, fue también la más abundante en cada uno de ellos (ver anexo). Por último puede señalarse un grupo formado por las especies Camponotus abdominalis, Myrmelachista sp. 1, Megalomyrmex sp. 1, Pheidole sp. 5, Hypoponera sp. 2, Pheidole sp. 6 y Pachycondyla carbonaria, las cuales estuvieron restringidos a lugares más recuperados y algunas solo al bosque maduro. Muestreos aislados realizados en otros lugares dentro de la reserva (alrededores de las instalaciones y otros bosques secundarios -categoría 0 en anexo) confirman la presencia de las especies Myrmelachista sp. 1 Megalomyrmex sp. 1, Hypoponera sp. 2 y Pheidole sp. 1 sólo en los tipos de bosque señalados.

\section{DISCUSIÓN}

La riqueza de hormigas registrada para La Planada (63 especies) es mucho mayor a la esperada para un bosque nublado a esta altura. Levings (1983) propone que la humedad y la escasa radiación solar, por causa de la neblina, son las condiciones responsables de la disminución de poblaciones de hormigas con la altitud. Estos dos fenómenos se dan ampliamente en La Planada, donde se calcula que hay una humedad relativa de $89 \%$ y alrededor de 887.5 horas de brillo solar al año, es decir un promedio de
2.4 horas/día, muy bajo si es comparado con un Bosque Tropical que recibe en promedio 6 o 7 horas/día (Bazzaz \& Pickett 1980). Esto lleva a pensar que son otros los factores que afectan más directamente la composición y diversidad de hormigas. Condiciones como temperatura, cobertura del suelo, complejidad estructural del bosque, composición y biomasa de artrópodos, entre otros, pueden determinar la cantidad y facilidad de encuentro de sustratos de nidificación y forrajeo (Greenslade \& Greenslade 1977, Wilson 1987, Andersen 1990).

Un posible factor que influye en esta riqueza es el mosaico de paisajes que posee La Planada. Al tener lugares con estadios sucesionales de diferentes edades se da la oportunidad que especies con diferentes requerimientos nutricionales y climáticos habiten allí y que estos bosques alberguen además de las especies especialistas, características de lugares poco perturbados, otras que sean generalistas, que son las que se encuentran con frecuencia en el suelo de zonas intervenidas (Greenslade \& Greenslade 1977, Molano 1994, Roth et al. 1994). Estos resultados hacen ver la necesidad de incluir dentro de las áreas protegidas, zonas heterogéneas que incluyan diversidad de ambientes (e.g. cobertura vegetal y tipos de suelo) para incrementar la diversidad de hormigas y otros organismos (Miller 1993, Roth et al. 1994, Lawton et al. 1998). Arens y Sánchez 
(1994) llegan a una conclusión similar en su investigación sobre los helechos arbóreos del género Cyathea de La Planada.

En este trabajo como en el de Belshaw y Bolton (1994) no se encontró una relación entre la edad del bosque y el número de especies de hormigas como la hallada en los estudios de Room (1975), Majer (1983), Majer y CamerPesci (1991) y Bustos y Ulloa-Chacón (19961997). Los lugares de muestreo no presentaron diferencias estadísticamente significativas en la riqueza de especies entre bosques con diferentes grados de perturbación. Los lugares con el mayor número de especies corresponden al pastizal $(\mathrm{Pa})$ y el bosque entresacado $(\mathrm{E})$, con valores de 25 y 26 respectivamente. El bosque de 10 años (10b) presentó el número más bajo de especies (12) en las dos fases de campo (anexo). Esto posiblemente se explique porque éste era un parche de bosque de menor tamaño que estaba cerca de un camino y que además corresponde a un estadio intermedio y muy homogéneo de la sucesión, en el cual se espera un aumento en la competencia entre especies pioneras y colonizadoras tardías (MacKay 1993).

Experimentos de campo han mostrado algunas condiciones que pueden asociarse al alto número de especies epígeas en áreas abiertas como pastizales y cultivos (Torres 1984a y 1984b). Torres (1984b) afirma que el elevado número de especies epígeas en estos lugares se debe, por una parte, a la presencia de cultivos con doseles cerrados que permiten la colonización de especies de bosque, y por otra, a que en estos sitios existe un mayor solapamiento alimenticio, gremios más compactos, un microclima más variable y niveles más altos de agresividad. Afirma además, que la mayor diversidad estructural del bosque actúa como un amortiguador reduciendo el número de microhábitats climáticos para las hormigas y por lo tanto reduciendo el número de especies en este estrato.

Majer y Beeston (1996) compararon la riqueza de especies entre lugares con diferentes tipos de perturbación y bosques control en el oeste de Australia y encontraron que los lugares de pastoreo mostraron la menor reducción en la riqueza comparado con campos agrícolas, caminos, zonas urbanas y minas.

La semejanza en la riqueza de especies entre estadios sucesionales de La Planada puede deberse a factores como los que explica Torres (1984b) o a los encontrados por Majer y Beeston (1996), sumados a la posibilidad de que con el aumento en la complejidad estructural del bosque, se creen también más sitios de anidación y forrajeo que están desplazados hacia el dosel (Greenslade \& Greenslade 1977, Majer \& Camer-Pesci 1991). Greenslade y Greenslade (1977) proponen que en condiciones de alta diversidad, especies más especializadas en el suelo desplazan hacia el dosel a aquellas especies más generalistas que habían dominado suelo y vegetación en estadios sucesionales de baja diversidad. En este trabajo el dosel no fue muestreado pero sin duda alberga muchas especies que no bajan nunca a estratos epígeos y por lo tanto no se registraron. Es probable, también, que el bajo número de especies encontradas en las áreas de bosque (si se espera un aumento gradual de la riqueza con el grado de recuperación) se deba a sesgos en los métodos de muestreo. El resultado puede ser sesgado porque el atún tiende a atraer hormigas que son generalistas, tales como las que prevalecen en hábitat modificados, y no ser tan efectivo en atraer especialistas, comunes en áreas menos perturbados (Greenslade \& Greenslade 1977, Levings 1983, Roth et al. 1994). Además, las hormigas tienden a ser nocturnas y crípticas en el bosque y diurnas en pastizales y cultivos (Greenslade \& Greenslade 1977, Torres 1984a), lo cual puede sesgar también el método de colección manual.

Los cambios en la composición de especies con el gradiente sucesional pueden atribuirse a los diferentes requerimientos alimenticios, de anidación y microclimáticos que las hormigas poseen (Bustos \& Ulloa-Chacón 1996-1997). Experimentos en campo muestran que la temperatura juega un importante papel en la distribución de hormigas porque determina sus actividades de forrajeo y lugares de anidación (Torres 1984b) y es un factor primordial en el desarrollo de larvas y pupas (Wilson 
1971). Se ha observado que especies de bosque resisten menos tiempo en altas temperaturas que las especies de áreas abiertas (Torres 1984b). Otros factores que influyen son la cantidad de hojarasca, la composición florística y la estructura del bosque que hacen que se formen nuevos microhábitats donde las hormigas pueden vivir (Levings 1983). En la Planada se observaron diferencias en algunas de estas variables en los diferentes estadios de la sucesión lo que puede explicar, al menos en parte, la variación de la composición de hormigas.

La diferencia en composición de especies de hormigas entre el bosque maduro (M) y los otros lugares de estudio (Fig. 2), muestra como una perturbación, así aparentemente no sea muy severa (como la extracción de madera), puede alterar el equilibrio que guardan las especies que habitan bosques primarios, resaltando la importancia de protegerlos. Greenslade y Greenslade (1977) obtuvieron un resultado similar, argumentando que después de una perturbación, aunque la vegetación se haya estabilizado, las hormigas siguen en un estado temprano de ajuste.

La fauna de hormigas de La Planada cumplió muchas de las características planteadas por Majer (1983), Brown (1991) y Pearson (1994) necesarias para ser buena bioindicadora. Estas características fueron preferencia y fidelidad a ciertas condiciones ambientales, gran abundancia, alta riqueza de especies, facilidad en el muestreo, manipulación e identificación.

Las hormigas de La Planada podrán ser utilizadas posteriormente en análisis de monitoreo de la recuperación de este bosque utilizando dos parámetros. El primero se refiere a las especies y grupos de especies que estuvieron restringidas a estadios sucesionales definidos (Cuadro 3), y el segundo corresponde a los índices de similaridad. Se propone la utilización del coeficiente de asociación Simple Matching, que tiene en cuenta la ausencia-presencia de las especies, porque con él las especies de hormigas relacionaron de forma muy satisfactoria los sitios con respecto a su grado de recuperación. Se descarta la utilización de pará- metros como riqueza, diversidad y equitabilidad de hormigas, dado que no se observaron diferencias de estos entre los sitios de muestreo.

\section{AGRADECIMIENTOS}

Este trabajo fue financiado por el Proyecto Biopacífico y por el programa de becas de la Fundación John y Catherine MacArthur, a través de La Reserva Natural la Planada y la Fundación FES. Gracias a Orlando Martínez (Universidad Nacional de Colombia) por su ayuda en el análisis estadístico, Edgard Palacio (Universidad Javeriana) por la determinación de parte del material de hormigas y a Humberto Mendoza (Instituto Humboldt) por la determinación del material vegetal. A Catalina Saravia (FEN) y todo el personal de La Planada por su colaboración en el trabajo de campo y a Carlos Valderrama, Carlos Arturo Mejia (Universidad de los Andes), Benedito Cortés L. y un revisor anónimo por sus comentarios al manuscrito.

\section{RESUMEN}

Para evaluar el uso de las hormigas como indicadores ecológicos del grado de recuperación del bosque en la Reserva Natural La Planada (Nariño, Colombia), se colectaron hormigas entre Febrero y Agosto de 1995 en siete lugares correspondientes a un gradiente sucesional: potrero, pastizal, dos bosques de 10 años y uno de $20 \mathrm{y}$ bosques entresacado y maduro. Se encontraron 63 especies de hormigas ( 29 géneros), con una riqueza entre 20 y 26 especies por lugar de estudio, excepto uno de los bosques de 10 años (12 especies). No se hallaron diferencias estadísticamente significativas en la riqueza y diversidad de especies entre los muestreos ni entre los lugares de estudio. Sin embargo, la composición de especies de hormigas si cambió entre los diferentes estadios sucesionales y los discriminó y asoció al analizarla con el índice de similaridad Simple Matching. Se propone la utilización de las hormigas en planes de monitoreo en La Planada utilizando la composición de especies como parámetro indicador.

\section{REFERENCIAS}

Andersen, A. N. 1990. The use of ant communities to evaluate change in Australian terrestrial ecosystems: A review and a recipe. Proc. Ecol. Soc. Aust. 16: 347-357. 
Anónimo. 1977. Zonas de vida o formaciones vegetales de Colombia. Memoria explicativa sobre el mapa ecológico. Vol. XIII, No 11. Instituto Geográfico Agustín Codazzi (IGAC), Santafé de Bogotá, 201 p.

Anónimo. 1986. Programa de los Andes Tropicales. Protegiendo un gran centro mundial de diversidad biológica. Fondo Mundial para la Naturaleza (WWF), Cali, $32 \mathrm{p}$.

Arens, N. C. \& P. Sánchez. 1994. Distribución de helechos arbóreos dentro del mosaico sucesional en un Bosque Nublado, Reserva Natural La Planada, Nariño, p. 18. In J. Cavelier \& A. Uribe (eds.). Resúmenes del Simposio Nacional "Diversidad Biológica, Conservación y Manejo de los Ecosistemas de Montaña en Colombia", Universidad de los Andes, Santafé de Bogotá, Colombia. Junio 6-10.

Bazzaz, F. A. \& S. Pickett. 1980. Physiological ecology of tropical succession: a comparative review. Ann. Rev. Ecol. Syst. 11: 287-310.

Belshaw, R. \& B. Bolton. 1994. A survey of the leaf litter ant fauna in Ghana, west Africa (Hymenoptera: Formicidae). J. Hym. Res. 3: 5-16.

Brown, K. S. 1991. Conservation of neotropical environments: insects as indicators, p. 349-404. In N. M. Collins \& J. Thomas (eds.). Conservation of insects and their habitats. Academic, San Diego.

Bustos, J. \& P. Ulloa-Chacón. 1996-1997. Mirmecofauna y perturbación en un bosque de niebla neotropical (Reserva Natural Hato Viejo, Valle del Cauca, Colombia). Rev. Biol. Trop. 44/45: 259-266.

Crisci, J. V. 1983. Introducción a la teoría y práctica de la taxonomía numérica. Monografía $\mathrm{N}^{\mathrm{o}} 26$ de biología. Secretaría General de la Organización de Estados Americanos. Programa Regional de Desarrollo Científico y Tecnológico, Washington D. C., 132 p.

Greenslade, P. J. M. \& P. Greenslade. 1977. Some effects of vegetation cover and disturbance on a tropical ant fauna. Insect. Soc. 24: 163-182.

Holloway, J. D. \& N. E. Stork. 1991. The dimensions of biodiversity: the use of invertebrates as indicators of human impact, p. 37-62. In D. L. Hawksworth (ed.). The biodiversity of microorganisms and invertebrates: its role in sustainable agriculture. Cab International, Wallingford, Inglaterra.

Janzen, D. H. 1987. Insect diversity of a Costa Rican Dry Forest: why keep it, and how?. Biol. J. Linn. Soc. 30: 343-356.
Kim, K. C. 1993. Biodiversity, conservation and inventory: why insects matter. Biodiv. Conserv. 2: 191214.

Kremen, C., R. Colwell, T. Erwin, D. Murphy, R. Noss \& M. Sanjayan. 1993. Terrestrial arthropod assemblages: their use in conservation planning. Conserv. Biol. 7: 796-808

Landres, P., J. Verner \& J. Thomas. 1988. Ecological uses of vertebrate indicator species: a critique. Conserv. Biol. 2: 316-328.

Lawton, J. H., D. E. Bignell, B. Bolton, G. F. Bloemers, P. Eggleton, P. M. Hammond, M. Hodda, R. D. Holt, T. B. Larsen, N. A. Mawdsley, N. E. Stork, D. S. Srivastava \& A. D. Watt. 1998. Biodiversity inventories, indicator taxa and effects of habitat modification in tropical forest. Nature 391: 72-75.

Levings, S. 1983. Seasonal, annual and among-site variation in the ground ant community of a deciduous Tropical Forest: some causes of patchy species distributions. Ecol. Monogr. 53: 435-455.

Ludwing, J. A. \& J. F. Reynolds. 1988. Statistical ecology: a primer on methods and computing. Wiley, Nueva York, $325 \mathrm{p}$.

Mackay, W. P. 1993. Succession of ant species (Hymenoptera: Formicidae) on low-level nuclear waste sites in northern New Mexico. Sociobiology 23: 1-11.

Magurran, A. E. 1988. Ecological diversity and its measurement. Princeton University, Nueva Jersey, 179 p.

Majer, J. 1983. Ants: bio-indicators of minesite rehabilitation, land-use, and land conservation. Environm. Manage. 7: 375-383.

Majer, J. \& P. Camer-Pesci. 1991. Ant species in tropical Australian tree crops and native ecosystems - is there a mosaic?. Biotropica 23: 173-181.

Majer, J. \& G. Beeston. 1996. The biodiversity integrity index: an illustration using ants in western Australia. Conserv. Biol. 1: 65-73.

Miller, J. C. 1993. Insect natural history, multi-species interaction and biodiversity in ecosystems. Biodiv. Conserv. 2: 233-241.

Molano, A. 1994. Hormigas (Hymenoptera: Formicidae) del bosque seco tropical y de agrosistemas de la región de Zambrano, Bolivar. Tesis de Pregrado, Universidad Nacional de Colombia, Santafé de Bogotá, 227 p. 
Ortiz von Halle, B. 1991. La fauna de los Bosques Montanos, p. 120-148. In: H. C. Uribe (ed.). Bosques de Niebla de Colombia. Banco de Occidente, Cali, Colombia.

Pearson, D. 1994. Selecting indicator taxa for the quantitative assessment of biodiversity. Phil. Trans R. Soc. Lond. B. 345: 75-79.

Romero, H. \& K. Jaffé. 1989. A comparison of methods for sampling ants (Hymenoptera, Formicidae) in savannas. Biotropica 21: 348-352.

Room, P. M. 1975. Diversity and organization of the ground foraging ant faunas of forest grassland and tree crops in Padua New Guinea. Austral. J. Zool. 23: 71-89.

Roth, D. S., I. Perfecto \& B. Rathcke. 1994. The effects of management systems on ground-foraging ant diversity in Costa Rica. Ecol. Appl. 4: 423-436.
Samways, M. J. 1993. Insects in biodiversity conservation: some perspectives and directives. Biodiv. Conserv. 2: 258-282.

Torres, J. A. 1984a. Niches and coexistence of ant communities in Puerto Rico: repeated patterns. Biotropica 16: 284-295.

Torres, J. A. 1984b. Diversity and distribution of ant communities in Puerto Rico. Biotropica 16: 296-303.

Wilson, E. O. 1971. The insect societies. Harvard University, Cambridge, Massachussetts 548 p.

Wilson, E. O. 1987. The arboreal ant fauna of Peruvian Amazon Forest: a first assessment. Biotropica 19: 245-251. 


\section{APENDICE 1}

Hormigas halladas en La Planada. Los números representan la abundancia de hormigas colectadas en trampas. La abundancia máxima es 96 (44 trampas epígeas, 44 arbóreas y 8 hipógeas). Los * representan las especies colectadas manualmente. P: potrero, Pa: pastizal, 10a y 10b: bosques de 10 años, 20: bosque de 20 años, E: bosque entresacado, M: bosque maduro y $O$ : lugares cercanos a las instalaciones y bosques cercanos donde se realizaron muestreos ( $p$, perturbados y bs, bosque secundario). 1 y 2 representan la primera y segunda fase de campo

Subfamilia/especie

MYRMICINAE

Acromyrmex sp.

Atta sp. (Reina) (1)

Crematogaster sp.

Cyphomyrmex castagnei

Lenomyrmex mandibularis

Megalomyrmex sp. 1

M. sp. 2

M. sp. 3

Monomorium sp. ${ }^{(1)}$

Neostruma sp.

Pheidole sp. 1

Ph. sp. 2

Ph. sp. 3

Ph. sp. 4

Ph. sp. 5

Ph. sp. 6

Ph. sp. 7

Ph. sp. $8^{(2)}$

Ph. sp. 9

Ph. sp. 10

Ph. sp. 11

Ph. sp. 12

Ph. sp. 13

Ph. sp. 14

Ph. sp. 15

Ph. sp. 16

Ph. sp. 17

Procryptocerus sp.

Rogeria sp. 1

Rogeria sp. 2

Solenopsis sp. grupo fugax

Solenopsis sp. 1

Stenamma felixi

PONERINAE

Gnamptogenys sp. 1

Gn. sp. 2

Hypoponera sp. 1

Hypoponera sp. 2

Pachycondyla apicalis

P. carbonaria

P. eleonorae

$P$. ferruginea

P. becculata

P. chyzeri

P. constricta

Simopelta sp. grupo willamsi
Sitios de muestreo
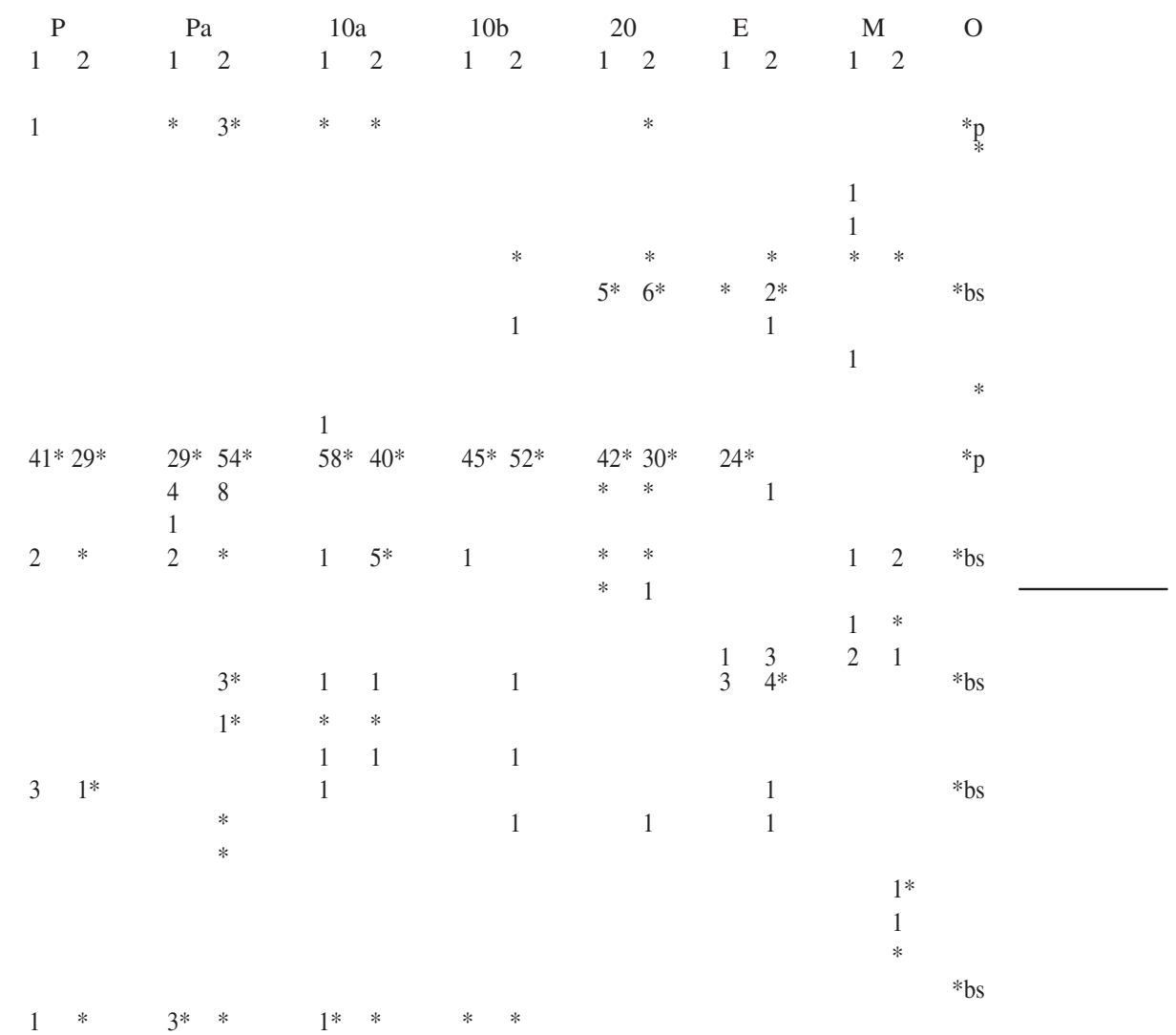

2

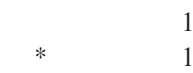

$3^{*} \quad 2$

1

$3 * \quad 2 *$

$4^{*} \quad 5^{*}$

3* 4*

$5^{*} 6^{*}$

4* 1* *bs-p

* * *

$18 * 11 * 30 * 30 *$

$15^{*} 23^{*} \quad 8^{*} \quad 8^{*}$ *bs

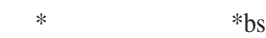

$28 * 34 *$

11 
Subfamilia/especie

\begin{tabular}{cccccccccccc}
\multicolumn{1}{c}{$\mathrm{P}$} & \multicolumn{1}{c}{$\mathrm{Pa}$} & \multicolumn{1}{c}{$10 \mathrm{a}$} & $10 \mathrm{~b}$ & 20 & $\mathrm{E}$ & $\mathrm{M}$ & $\mathrm{O}$
\end{tabular}

ECITONINAE

Nomamyrmex hartigi

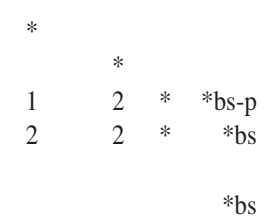

Cheliomyrmex andicolus

Eciton lucanoides

Labidus praedator

L. coecus

Neivamyrmex sp.

FORMICINAE

Brachymyrmex sp.

Camponotus abdominalis

C. sp. 1

C. sp. 2

Myrmelachista sp. ${ }^{(3)}$

M. sp. $2^{(4)}$

Paratrechina sp.

DOLICHODERINAE

Azteca sp.

Dorymyrmex sp. 1

D. sp. 2

Linepithema sp. 1

L. sp. 2

Total

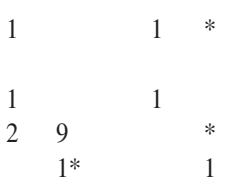

1*

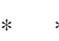

3

*

*bs

*bs

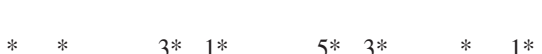

1

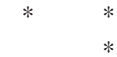

$*$

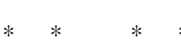

$*$

*bs-p

$\begin{array}{llllll}* & * & 1 & 2 * & 1 & 1\end{array}$

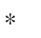

$5 *$

* *

1

*bs-p

$3 *$

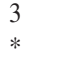

2* $1 *$

2
1

$*$

$2 * 1 *$

22

25

20

12

$6^{*}$

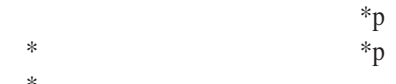

26

22

22

(1) Hormigas encontradas en la cocina de La Planada.

(2) En el bosque entresacado (E) esta hormiga fue encontrada dentro del tallo del arbusto Drymonia sp. (Gesneriaceae) y Palicourea sp. 4 (Rubiaceae) (colección Humberto Mendoza).

(3) Hormiga encontrada entre el tronco de Nectandra sp. y Aniba sp. (Lauraceae).

(4) Encontrada dentro del tronco de Eschweilera sp. (Lecythidaceae) y en Roagea glabra (Meliaceae). 